\title{
Stage IIA Gestational Trophoblastic Tumor AJCC v7
}

National Cancer Institute

\section{Source}

National Cancer Institute. Stage IIA Gestational Trophoblastic Tumor A/CC v7. NCI

Thesaurus. Code C87154.

Stage IIA includes: T2, M0, Risk factors: low risk. T2: Tumor extends to other genital structures (ovary, tube, vagina, broad ligaments) by metastasis or direct extension. M0: No distant metastasis. (AJCC 7th ed.) 